\title{
\begin{tabular}{l|l} 
Mibraries & DSpace@MIT
\end{tabular}
}

\author{
MIT Open Access Articles
}

Sensitization of silicon by singlet exciton fission in tetracene

The MIT Faculty has made this article openly available. Please share how this access benefits you. Your story matters.

Citation: Wu, Tony C. et al. "Sensitization of silicon by singlet exciton fission in tetracene",

Physical Chemistry of Semiconductor Materials and Interfaces XIX, Proceedings of SPIE, 11464, Society of Photo-Optical Instrumentation Engineers, 2020, 1146416. (c) 2020 SPIE.

As Published: http://dx.doi.org/10.1117/12.2567365

Publisher: Society of Photo-Optical Instrumentation Engineers (SPIE)

Persistent URL: https://hdl.handle.net/1721.1/129729

Version: Final published version: final published article, as it appeared in a journal, conference proceedings, or other formally published context

Terms of Use: Article is made available in accordance with the publisher's policy and may be subject to US copyright law. Please refer to the publisher's site for terms of use. 


\section{Sensitization of silicon by singlet exciton fission in tetracene}

Wu, Tony, Einzinger, Markus, Kompalla, Julia, Smith, Hannah, Perkinson, Collin, et al.

Tony C. Wu, Markus Einzinger, Julia Kompalla, Hannah Smith, Collin Perkinson, Leas Nienhaus, Sarah Wieghold, Daniel Congreve, Nicholas Thompson, Antoine Kahn, Moungi Bawendi, Marc A. Baldo, "Sensitization of silicon by singlet exciton fission in tetracene," Proc. SPIE 11464, Physical Chemistry of Semiconductor Materials and Interfaces XIX, 1146416 (20 August 2020); doi: 10.1117/12.2567365

SPIE. Event: SPIE Nanoscience + Engineering, 2020, Online Only 


\title{
Sensitization of silicon by singlet exciton fission in tetracene Tony $\mathrm{C} \mathrm{Wu}{ }^{* a}$, Markus Einzinger*a, Julia Kompalla ${ }^{\mathrm{a}}$, Hannah Smith ${ }^{\mathrm{b}}$, Collin Perkingson ${ }^{\mathrm{a}}$, Leas Nienhaus ${ }^{\mathrm{a}}$, Sarah Wieghold ${ }^{\mathrm{a}}$, Daniel Congreve ${ }^{\mathrm{a}}$, Nicholas Thompson ${ }^{\mathrm{a}}$, Antoine Kahn ${ }^{\mathrm{b}}$, Moungi Bawendi $^{\mathrm{a}}$, Marc A Baldo ${ }^{\mathrm{a}}$ \\ ${ }^{a}$ Massachusetts Institute of Technology, 77 Massachusetts Ave, Cambridge, MA USA 02139; \\ ${ }^{\mathrm{b}}$ Princeton University, Princeton, NJ USA 08544
}

\begin{abstract}
Singlet fission can split a high energy singlet exciton and generate two lower energy triplet excitons. This process has shown near 200 percent triplet exciton yield. Sensitizing solar cells with singlet fission material, it can potentially increase the power conversion efficiency limit from 29 percent to 35 percent. Singlet fission in the tetracene is known to be efficient, and the energy of the triplet excitons are energetically matched to the silicon bandgap. In this work, we designed an optical measurement with an external magnetic field to determine the efficiencies of triplet exciton transfer from tetracene to silicon. Using this method, we have found that a passivation layer of 8 angstroms of hafnium oxynitride on silicon allows efficient triplet exciton transfer around 133 percent.
\end{abstract}

Keywords: Singlet Fission, Hybrid Silicon Solar Cell

\section{INTRODUCTION}

Our main energy source is burning fossil fuels. Solar energy is a clean, alternate energy source. In this work, we will utilize singlet fission to increase solar cell power conversion efficiency.

\subsection{Singlet fission}

Singlet fission is a mechanism found in some organic materials, that a high energy singlet exciton could generate two lower energy triplet excitons. Figure 1a shows the schematic of singlet fission. Due to energy conservation, the triplet state energy $E_{T}$ needs to be about half of the singlet state energy $E_{S}$ for an efficient singlet fission to occur. The singlet exciton state first transitions into a triplet-triplet (TT) pair state ${ }^{1}$. This TT pair state can then dissociate into two individual triplet excitons, effectively achieving energy down conversion. This down conversion process can be efficient because direct transition from singlet to triplet state is not allowed or inefficient due to selection rules. However, the TT pair state is a two particle system with a mixture of 9 possible states. Among the TT pair states, there are some pair states that have singlet state characteristics. Therefore, this transition from singlet states to TT pair states and then to two tri plet excitons are allowed. In fact, there have been multiple studies showing efficient singlet fission rates in different organic materials ${ }^{2}$.

\subsection{Down conversion to overcome the Shockley-Queisser limit}

In a perfect single junction solar cell, the theoretical maximum power conversion efficiency (PCE) is around $33.7 \%{ }^{3}$. This is mainly due to the trade-off between thermalization loss for photons collected above bandgap and absorption loss below bandgap. With a down converter, that converts high energy excitons/photons $2 \mathrm{E}$ to two lower energy excitons/photons E, a solar cell can extract extra energy from thermalization loss. Figure $1 \mathrm{~b}$ shows the breakdown of extra energy collected for solar cells with down conversion. In the blue area, the solar cell could utilize about twice more photocurrent where the spectrum $\mathrm{E}_{\mathrm{hv}}>2 \mathrm{E}_{\mathrm{g}}$. In this case, a $\mathrm{Eg}=1.14 \mathrm{eV}$ solar cell collects about $33.4 \%$ energy from the spectrum, where down conversion can collect another additional $7.8 \%$, giving the possibility of overcoming the Shockley-Queisser limit. In a silicon solar cell, the maximum intrinsic efficiency can be further limited to around $29 \%$ due to non-radiative Auger recombination ${ }^{4}$. Therefore, a silicon solar cell utilizing perfect down conversion has a theoretical maximum $35 \% \mathrm{PCE}^{5}$.

Physical Chemistry of Semiconductor Materials and Interfaces IX, edited by Christian Nielsen,

Daniel Congreve, Andrew J. Musser, Proc. of SPIE Vol. 11464, 1146416 · C 2020

SPIE $\cdot$ CCC code: $0277-786 \mathrm{X} / 20 / \$ 21 \cdot$ doi: $10.1117 / 12.2567365$ 


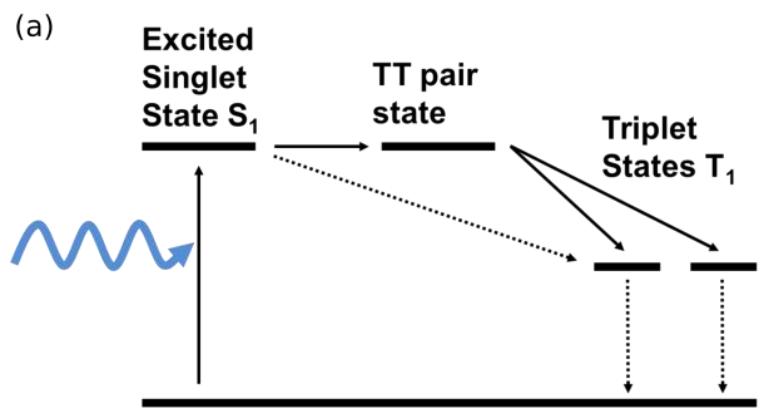

Ground Singlet State $S_{0}$

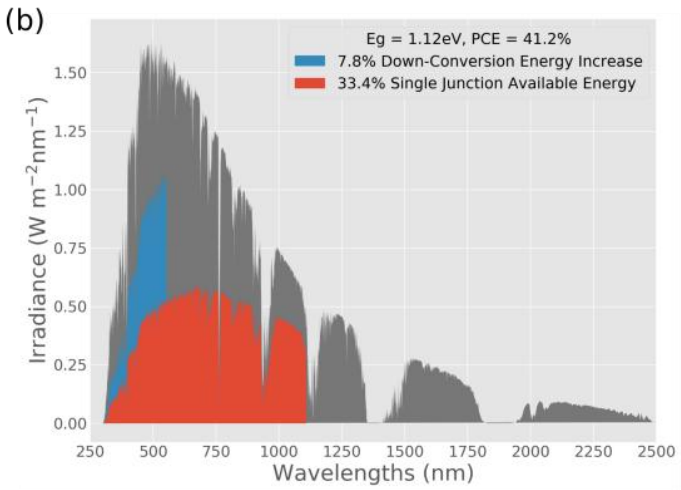

Figure 1. (a) Schematics of singlet fission. Singlet exciton $S_{1}$ first transitions to a TT pair state and then splits into two triplet exciton states $T_{1}$. Direct transition from $S_{1}$ to $T_{1}$ is inefficient. (b) Efficiency of silicon solar cell with down conversion. This breakdown shows an additional $7.8 \%$ of extra energy collected with down conversion on an $\mathrm{E}_{\mathrm{g}}=1.12 \mathrm{eV}$ solar cell.

\subsection{Magnetic field effect in singlet fission rates}

An external magnetic field can affect the singlet fission rates. This is particularly useful for identifying singlet fission as well as assisting the characterization of fission efficiencies. The magnetic field effect (MFE) of singlet fission rate comes from the modification of the amount of TT states that have singlet characteristics. As shown in figure 1a, TT states assists the transition from a singlet exciton to two triplet excitons. The fission rate first increases with magnetic field $(0 \sim 0.03 \mathrm{~T})$ due to increasing TT pair states (3 to 6) with singlet characteristics. TT pair states then decrease to 2 states with singlet characteristics at high magnetic fields $(0.03 \mathrm{~T} \rightarrow 0.5 \mathrm{~T})$, thus slowing the fission rate.

The singlet fission MFE gives a distinctive dependency. In figure 2a, the triplet population MFE follows the fission rate because slower fission rates result in less triplet excitons. However, the singlet population MFE is oppo site where a slower fission rate results in more singlet excitons. These assists identification of singlet fission in organic materials. For example in figure $2 b$, the green and red shows the fluorescence change of the tetracene with magnetic fields, which follows the trend of singlet population. The blue curve shows the photocurrent change in a tetracene photovoltaic device. This shows the photocurrent are mainly collected from triplet excitons.
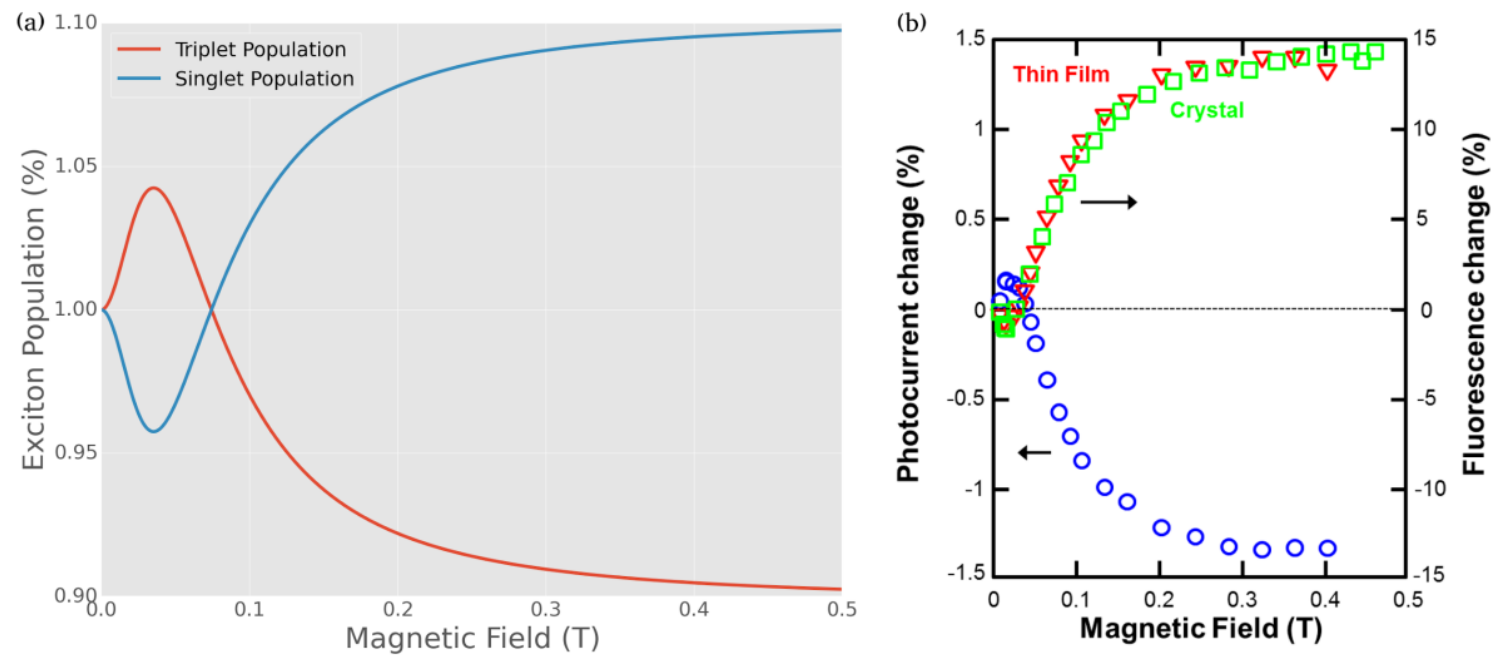

Figure 2. (a) Simulated singlet and triplet population change under external magnetic fields. (b) MFE of fluorescence change in tetracene thin film (red) and crystals (green). MFE of photocurrent change in tetracene devices (blue) ${ }^{6}$. 


\section{SINGLET-FISSION EFFICIENCY IN TETRACENE}

In this work, we will be using tetracene as the main singlet fission material for sensitizing silicon solar cells. Several organic materials show efficient fission efficiencies such as pentacene, which has internal quantum efficiencies of $160 \%$ and $200 \%$ triplet exciton yields. However, pentacene triplet energy is $\mathrm{E}_{\mathrm{T}}=0.86 \mathrm{eV}^{7,8}$, which makes it unsuitable as the down converter for silicon solar cells. On the other hand, tetracene triplet energy is $\mathrm{E}_{\mathrm{T}}=1.25 \mathrm{eV}$, slightly above the silicon bandgap, making it an attractive singlet fission candidate for silicon solar cells. In tetracene, the fission process is thought to be slightly endothermic with singlet energy $E_{S}=2.3 \mathrm{eV}^{9}$. However, the yield of triplet excitons can be aided by spin conservation, which eliminates a thermalization loss pathway. In this section, we will focus on characterizing singlet fission efficiencies in tetracene.

\subsection{Internal quantum efficiency of tetracene photovoltaics}

Singlet exciton fission in photovoltaic devices is complicated by the presence of additional important loss pathways such as singlet exciton dissociation into charges. Practical characterization of singlet fission requires measurements of the triplet yield in devices. A lower limit for this yield can be defined by the internal quantum yield (IQE), which is the ratio of charges generated in the cell to photons absorbed for the specific layer. In a tetracene device, determination of the IQE is especially challenging, since its optical absorption overlaps with the usual acceptor material $\mathrm{C}_{60}$.

The tetracene photovoltaic devices are fabricated with a PEDOT:PSS layer spin-coated on a cleaned ITO glass substrate. Then, m-MTDATA $(8 \mathrm{~nm})$, tetracene $(x \mathrm{~nm})$, C60 $(30 \mathrm{~nm})$, BCP $(15 \mathrm{~nm})$ and silver $(100 \mathrm{~nm})$ are deposited onto the substrate subsequently with thermal evaporation. For thinner tetracene layer devices, we build photodetector structures with multiple pairs of tetracene/ $\mathrm{C}_{60}$ layers. To determine the IQE of tetracene in a solar cell device from external quantum efficiency (EQE), we need to calculate the absorption of each layer in the device to calculate the contributions. For each material, the optical $n$ and $k$ parameters are measured from wavelength dependent transmissions and reflections. With an optical transfer matrix ${ }^{10}$, we can estimate each layer's absorption spectra in the device, fitting the overall wavelength dependent $\mathrm{EQE}$ by separately adjusting the respective IQEs of all layers, mainly tetracene and $\mathrm{C}_{60}$.

To find the optimal, we measure the IQEs of different tetracene devices, and obtain a maximum IQE of $127 \pm 18 \%$ with tetracene layer thickness $x=25 \mathrm{~nm}$. This optimal thickness is a balance between a thicker tetracene layer for more triplet exciton generation and a thinner layer for better collection efficiency. The IQE dependency on tetracene thickness is shown in figure $3 \mathrm{~d}$. Alongside is the comparison to the IQE of pentacene devices, which shows a better IQE of $160 \%$ at a thinner layer due to faster singlet fission rates.
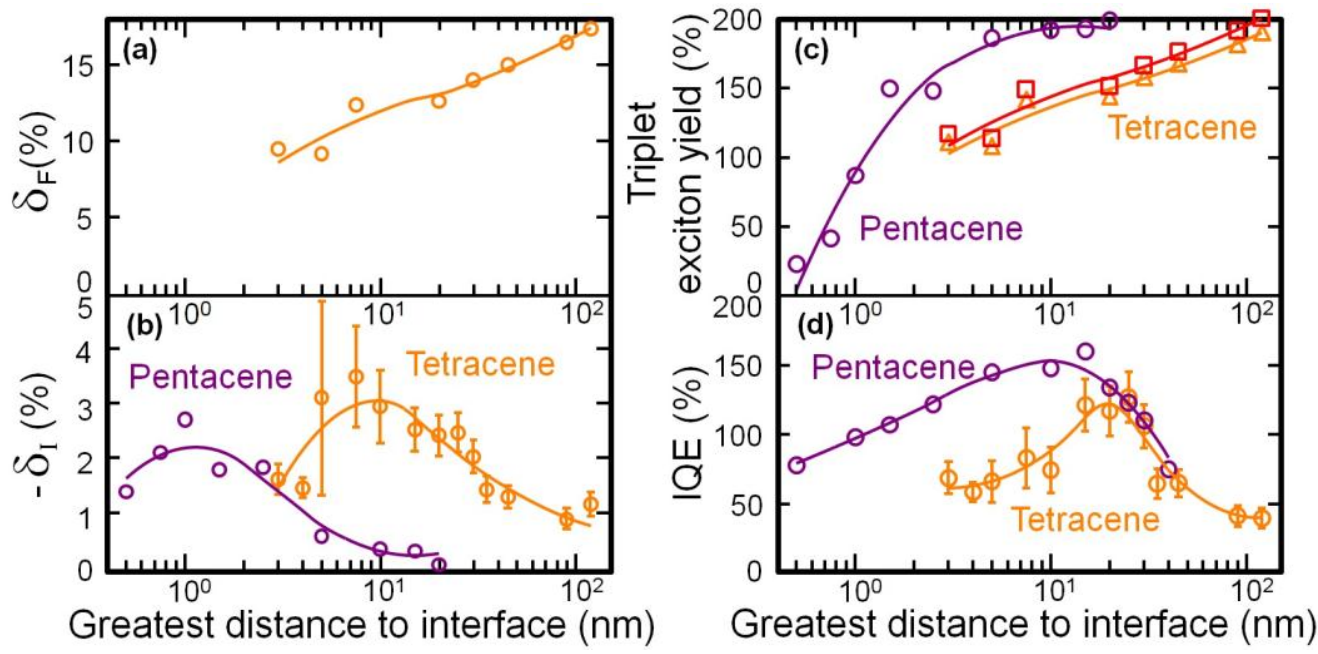

Figure 3. (a) and (b) are the fluorescence change $\delta_{\mathrm{F}}$ and photocurrent change $\delta_{\mathrm{I}}$ under a magnetic field $(>0.4 \mathrm{~T})$ in both tetracene and pentacene. The fluorescence changes are measured in the same tetracene $/ \mathrm{C}_{60}$ structure to the photocurrent devices. (c) Tetracene and pentacene triplet exciton yields are modeled from fluorescence and photocurrent changes. (d) The IQEs of thin layers of pentacene and tetracene reflect the changes in triplet yield, and triplet diffusion losses in thick films. 


\subsection{Using magnetic field effect to characterize triplet yields}

The MFE can also provide a quantitative model of the triplet exciton yield of tetracene. In figure 3ab, we plot the fluorescence and photocurrent change under large magnetic fields $(>0.4 \mathrm{~T})$. The tetracene fluorescence change here is measured on a thin film which consists of the same tetracene $/ \mathrm{C}_{60}$ layers without the other layers. The photocurrent changes are calculated into consideration of its corresponding tetracene IQEs in figure $3 \mathrm{~d}$.

To calculate triplet yields, we model the exciton dynamics in the devices with a singlet fission rate at 0 and high field $k_{f 0}$ and $k_{f B}$, singlet exciton dissociation rate $k_{S}(d)$ which depends on distance, and radiative rate $k_{R}$. For simplicity, we normalize all rates to $k_{f 0}$, which gives us high field fission rate $\chi_{f B}$, singlet dissociation rate $\chi_{s}(d)$ and radiative rate $\chi_{R}$. With these rates, we can deduct the fluorescence and photocurrent change as,

$$
\begin{gathered}
\delta_{F}(d)=\frac{1-\chi_{f B}}{\chi_{S}(d)+\chi_{f B}+\chi_{R}} \\
\delta_{I}(d)=\frac{\left(\chi_{f B}-1\right)\left(\chi_{S}(d)+2 \chi_{R}\right)}{\left(\chi_{S}(d)+\chi_{f B}+\chi_{R}\right)\left(\chi_{S}(d)+2\right)}
\end{gathered}
$$

Fitting the two equations (1) and (2) to $\delta_{F}$ and photocurrent change $\delta_{I}$ in figure $3 \mathrm{ab}$, we can solve all the rates and singlet dissociation rate $\chi_{s}(d)$ at each tetracene thickness. This can then calculate the triplet yields with different tetracene devices which is shown in figure $3 \mathrm{c}$. We can see that the tetracene triplet exciton yields are close to $200 \%$ for thick tetracene layers. However, as a comparison to pentacene, the tetracene triplet generation is much slower. For our optimal IQE device, the triplet yield is $153 \pm 5 \%$.

\section{TRIPLET EXCITON TRANSFER TO SILICON}

From section 2, tetracene is a potential down conversion material for silicon, due to both triplet exciton energy matching and sufficient single fission efficiencies. To study whether the triplet excitons transfer to the silicon, we directly measure the PL emission from silicon. Figure 4a shows a schematic on how the triplets generated in the tetracene layer can sensitize the silicon substrate. There are two possible effects that triplet excitons can increase silicon PL. Electric-fieldeffect passivation (orange charges) is caused by exciton dissociation at the interface or charge diffusion from the silicon. Additionally, electron-hole pairs in silicon can also be created by singlet exciton fission in tetracene (green) and energy transfer (light red) to silicon. In this study, we would like to identify triplet energy transfer directly to silicon, which is the effect that could overcome the Shockley-Queisser limit.

\subsection{Device structure}

Figure 4a shows a simple schematics of the device structure. The silicon wafers are first clean with the RCA protocol. The silicon surface is then passivated with atomic layer deposition with $\mathrm{HfO}_{\mathrm{x}} \mathrm{N}_{\mathrm{y}}$. After passivation, $30 \mathrm{~nm}$ of tetracene is thermally deposited onto the silicon substrate.

\subsection{Measurement setup}

To measure the magnetic field effect of the silicon emission, we use an infrared spectrometer instead of an IR detector to make sure that the photoluminescence comes from silicon emission. Figure $4 \mathrm{~b}$ is the setup to measure the MFE of the silicon emission spectrum. The samples are excited with a strong $532 \mathrm{~nm}$ laser and placed between a strong electromagnet. This setup is also modified to measure the excitation spectrum, which is the silicon emission with different excitation wavelengths. The magnet is removed and the excitation light source is changed to a SuperK. Because silicon PL efficiency is also dependent on excitation wavelength, we also measure the silicon excitation spectrum without tetracene as normalization and comparison. 

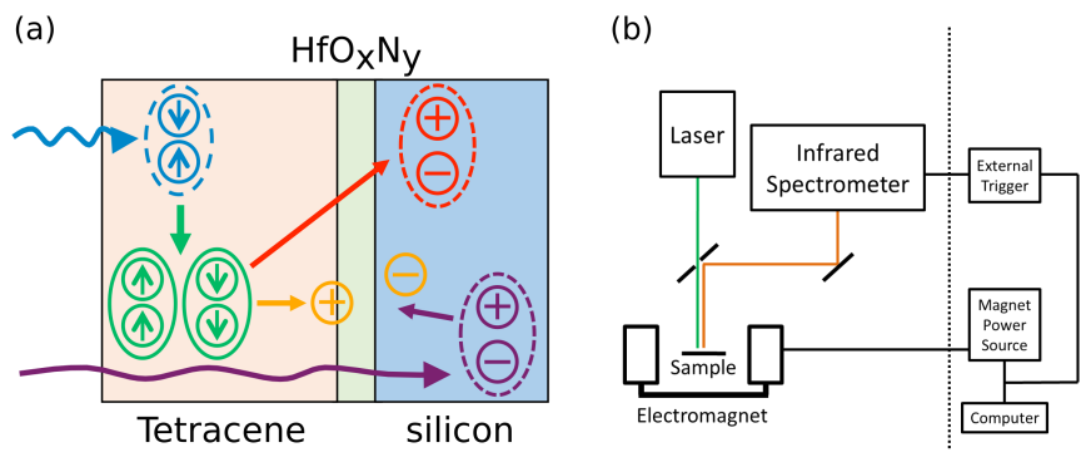

Figure 4. (a) Silicon substrate with singlet fission sensitization. (b) Spectrally resolved magnetic field effect measurement setup.

\subsection{Photoluminescence result}

The first indication of triplet exciton transfer is shown in figure 5a. The silicon emission first increases with small external magnetic fields and then decreases at large fields. This is the same trend as triplet MFE (figure 2a), which indicates that the silicon emissions are mediated by triplet excitons from tetracene.

In figure $5 \mathrm{~b}$, the excitation spectra also shows the increase of silicon PL in the tetracene absorption region for wavelengths $<550 \mathrm{~nm}$. The red and green dashed curves are the fitted silicon and tetracene absorption to the excitation spectra, which shows the tetracene absorption contribution in the silicon PL.
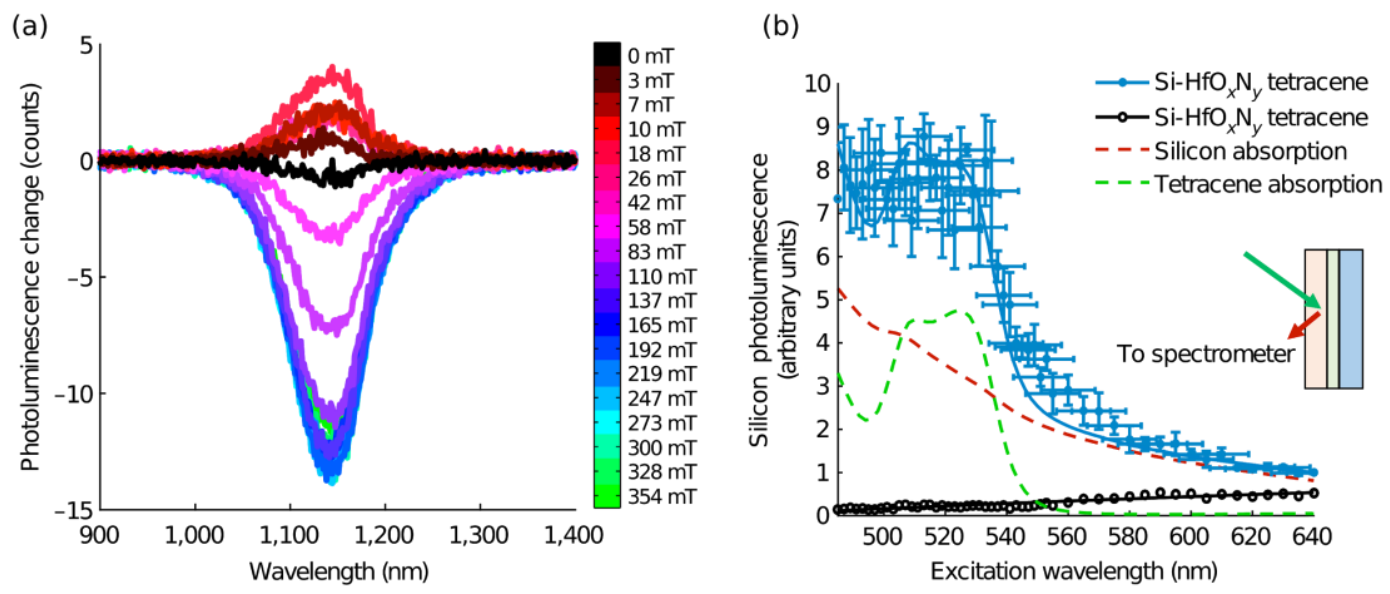

Figure 5. (a) Silicon spectrum changes with different magnetic fields. This spectral MFE is measured on a tetracene/HfO $\mathrm{N}_{\mathrm{y}}$ $(0.8 \mathrm{~nm}) /$ silicon device with $532 \mathrm{~nm}$ excitation. (b) The silicon emission excitation spectrum (blue) from the same device structure. The red and green curves are the fitted silicon and tetracene absorption contributions.

\subsection{Characterizing triplet exciton transfer}

However, due to the electric-field-effect passivation possibility (figure 4a, orange), the above result does not directly indicate the triplet excitons transferred to the silicon. To determine triplet exciton transfer, we need to measure the passivation effects and exciton transfer separately. Silicon PL comes from the electron-hole pairs generated in silicon. When exciting with $532 \mathrm{~nm}$, the triplet exciton generated in the tetracene can both affect the surface passivation and exciton transfer to increase the silicon PL. However, if we excite the sample with $785 \mathrm{~nm}$ at the same time with $532 \mathrm{~nm}$, the electron-hole pairs generated by this red laser will be affected more by the passivation effects.

In figure $6 \mathrm{a}$, we can see that the $532 \mathrm{~nm}+785 \mathrm{~nm}$, with red excitation intensity > green, the magnetic field effect is less than $532 \mathrm{~nm}$ excitation. This indicates that other than magnetic field effect from passivation, there is also triplet exciton transfer to increase the silicon PL change. 
Using the data from the $532 \mathrm{~nm}$ and $532 \mathrm{~nm}+785 \mathrm{~nm}$ excitation photoluminescence measurements that are shown in figure 6a, we employ a model to quantify the total exciton yields, which is the number of excitons transferred to silicon divided by the number of singlet excitons created in tetracene. By comparing the effects of the applied magnetic field on the photoluminescence of the samples, we obtain a peak total exciton yield of $133 \pm 13 \%$.

(a)

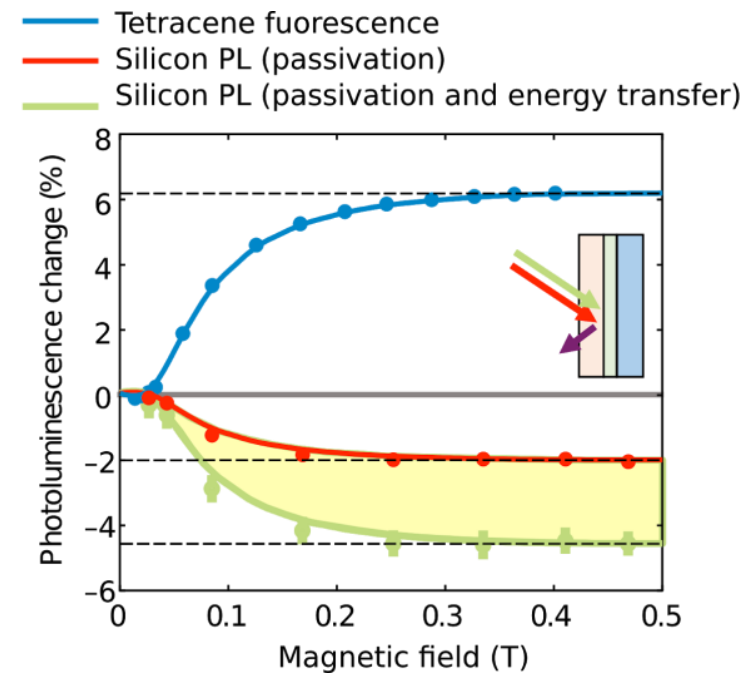

(b)

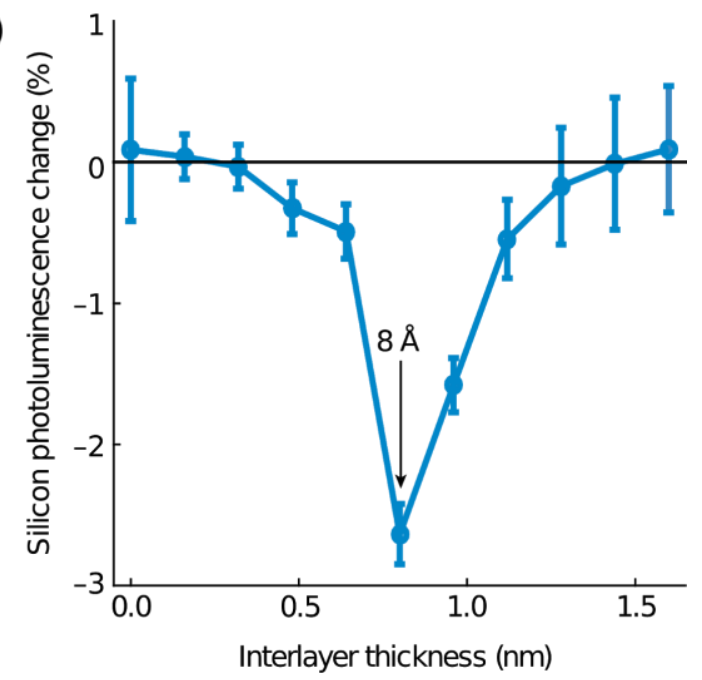

Figure 6. (a) The effect of the applied magnetic field on the photoluminescence of photoluminescent samples photoexcited at $\lambda=532 \mathrm{~nm}$. To quantify the electric-field-effect passivation we employ a second beam at $\lambda=785 \mathrm{~nm}$ that is sensitive to passivation changes but does not drive energy transfer from the tetracene. The solid lines are fit to the singlet-fission characteristic in the tetracene, as determined from the fluorescence. (b) Silicon PL MFE with respect to $\mathrm{HfO}_{\mathrm{x}} \mathrm{N}_{\mathrm{y}}$ thickness.

\subsection{Optimi zing passivation layer thickness}

We have also measured the MFE of the silicon PL with different $\mathrm{HfO}_{\mathrm{x}} \mathrm{N}_{\mathrm{y}}$ passivation thicknesses. The optimal thickness is at $0.8 \mathrm{~nm}$, shown in figure $6 \mathrm{~b}$. This is a tradeoff between the lack of surface passivation at the silicon surface, and blocking the triplet exciton for transferring into silicon. The fact that it is possible to turn this effect off by incre asing the interlayer thickness suggests the presence of a tunneling process.

\section{CONCLUSION}

This work paves the path for using tetracene as a singlet fission sensitizer for silicon solar cells. The down conversion layer in solar cells could help overcome the Shockley-Queisser limit and increase power conversion efficiencies. Here, we have determined efficient singlet fission efficiencies in tetracene with maximum IQE of $127 \%$. We also demonstrated triplet exciton from tetracene to silicon. The transfer was confirmed by designing a spectrally resolved MFE experiment. Using this method, we have found that a passivation layer of $0.8 \mathrm{~nm}$ of $\mathrm{HfO}_{\mathrm{x}} \mathrm{N}_{\mathrm{y}}$ on silicon allows efficient triplet exciton transfer around 133 percent.

The next step is to improve the efficiency of sensitized cells ${ }^{11}$, which is at present limited by poor carrier collection from the front surface of the silicon. Thinner silicon and tighter control over materials, processing and fabrication will increase efficiency, with a corresponding improvement in the ratio between the effects of the magnetic field on the photoluminescence and the photocurrent of the cell.

\section{REFERENCES}

[1] Merrifield, RE, Avakian, P and Groff, RP, "Fission of singlet excitons into pairs of triplet excitons in tetracene crystals," Chemical Physics Letters 3(6):386-388 (1969) 
[2] Congreve, D.N., Lee, J., Thompson, N.J., Hontz, E., Yost, S.R., Reusswig, P.D., Bahlke, M.E., Reineke, S., Van Voorhis, T. and Baldo, M.A., "External quantum efficiency above $100 \%$ in a singlet-exciton-fission-based organic photovoltaic cell," Science 340(6130) (2013)

[3] Shockley, W. and Queisser, H.J., "Detailed balance limit of efficiency of p- n junction solar cells," Journal of applied physics 32(3) (1961).

[4] Andreani, L.C., Bozzola, A., Kowalczewski, P., Liscidini, M. and Redorici, L., "Silicon solar cells: toward the efficiency limits," Advances in Physics: X 4(1) 2019.

[5] Rao, A. and Friend, R.H., "Harnessing singlet exciton fission to break the Shockley-Queisser limit," Nature reviews materials 2 (11) (2017).

[6] Wu, T.C., Thompson, N.J., Congreve, D.N., Hontz, E., Yost, S.R., Van Voorhis, T. and Baldo, M.A., "Singlet fission efficiency in tetracene-based organic solar cells," Applied Physics Letters 104(19) (2014).

[7] Geacintov, N.E., Burgos, J., Pope, M. and Strom, C., 1971. Heterofission of pentacene excited singlets in pentacene-doped tetracene crystals. Chemical Physics Letters, 11(4), (1971).

[8] Burgos, J., Pope, M., Swenberg, C.E. and Alfano, R.R., "Heterofission in pentacene- doped tetracene single crystals," physica status solidi (b) 83(1) (1977).

[9] Tomkiewicz, Y., Groff, R.P. and Avakian, P., "Spectroscopic approach to energetics of exciton fission and fusion in tetracene crystals," The Journal of Chemical Physics 54(10) (1971).

[10] Jung, S., Kim, K.Y., Lee, Y.I., Youn, J.H., Moon, H.T., Jang, J. and Kim, J., "Optical modeling and analysis of organic solar cells with coherent multilayers and incoherent glass substrate using generalized transfer matrix method," Japanese Journal of Applied Physics 50(12R) (2011).

[11] Einzinger, M., Wu, T., Kompalla, J.F., Smith, H.L., Perkinson, C.F., Nienhaus, L., Wieghold, S., Congreve, D.N., Kahn, A., Bawendi, M.G. and Baldo, M.A., "Sensitization of silicon by singlet exciton fission in tetracene," Nature 571(7763) (2019). 